\title{
EL DOBLE DE LA PALABRA. EL MITO DE ECO EN LA LITERATURA INGLESA
}

\section{CONTEMPORÁNEA}

\section{LAURA MONRÓS GASPAR UNIVERSITAT DE VALÈNCIA}

laura.monros@uv.es

Article received on 31.01.2011

Accepted on 25.05.2011

\begin{abstract}
RESUMEN
El objetivo de este artículo es el estudio de la figura de Eco en la literatura contemporánea escrita en lengua inglesa a partir de trabajos de A.S. Byatt, Timberlake Wertenbaker, Nicole Ward J ouve y Moniza Alvi entre otros. En poesía, teatro y relatos cortos, analizamos a Eco primero desde la literatura crítica publicada sobre la ninfa y posteriormente desde la perspectiva de oposiciones binarias uno/otro, metrópolis/colonia, palabra/silencio. Consideradas en conjunto, las obras revisadas en este artículo demuestran cómo la recepción del mito en la literatura contemporánea se encuentra estrechamente ligada a la evolución del concepto del doble en la cultura occidental.
\end{abstract}

PALABRAS CLAVE

Eco, recepción, literatura inglesa, doble.

\section{THE DOUBLE OF THE WORD. THE MYTH OF ECHO} IN CONTEMPORARY ENGLISH LITERATURE

\section{ABSTRACT}

This article explores the figure of Echo in contemporary English literature focusing on works by A.S. Byatt, Timberlake Wertenbaker, Nicole Ward Jouve and Moniza Alvi among others. After an introduction on the secondary literature published on the nymph, Echo is analyzed under the perspective of opposites such as self/other, metropolis/colony, word/silence in poetry, theatre and short stories. Considered en masse, the works discussed in this article manifest how the reception of Echo is closely linked to the concept of the double in Western cultures.

KEYWORDS

Echo, reception, English literature, double.

\section{PRELIMINARES: PRESENCIA DE ECO EN LA CULTURA OCCIDENTAL}

Numerosos estudios se han publicado en el último siglo acerca de la presencia de la figura de Eco en la cultura occidental. Todos ellos coinciden, a grandes rasgos y desde diferentes epígrafes, en dos ejes fundamentales en torno a los que se pueden englobar las reflexiones de recreaciones modernas del mito: la identidad del individuo y la dualidad de 
la voz y la palabra. Una breve aproximación a estos trabajos más recientes permite discernir la mirada que tanto desde la mitocrítica como desde la escritura de creación ha dirigido la recepción contemporánea del mito. Nuestra lectura en estas páginas se centra en las letras inglesas aunque por imperativos intrínsecos a la naturaleza misma de los estudios de recepción y de literatura comparada apoyaremos nuestra interpretación con textos críticos referidos también a otras tradiciones. El corpus de nuestro estudio en esta primera sección viene definido por la evolución del tratamiento del mito de Eco en los diferentes ámbitos de especialización considerados.

Dejando de lado los trabajos dedicados exclusivamente al estudio de la presencia de la ninfa en la literatura clásica greco-latina, más de una decena de monografías publicadas entre la segunda mitad del siglo XX y los comienzos del XXI ofrecen una visión bastante aproximada de la recepción de la figura de Eco en la literatura moderna y contemporánea. El volumen de Colby de 1920 es pionero en el análisis de la presencia de la ninfa en textos escritos en lengua inglesa. El legado del Colby es recogido en 1967 por Louise Vinge en The Narcissus Theme in Western European Literature up to the Early 19th Century, aunque todavía encontramos a Eco supeditada al análisis de su contrapunto ovidiano, Narciso, exponente por excelencia del estudio del doble en la literatura occidental desde el impulso freudiano.

El punto de inflexión en los estudios dedicados a la figura de Eco viene marcado a finales de la década de los setenta y comienzos de los ochenta por Marina Mizzau y J ohn Hollander en sus respectivos Eco e Narciso: Parole e silenzi nel conflitto uomo-donna y The Figure of Echo. A Mode of Allusion from Milton and After, publicados en 1979 y 1981. El ensayo de Mizzau bebe del feminismo francés de los setenta con Hélène Cixous y 'La risa de la Medusa' (1975) al abordar la cuestión del intercambio lingüístico entre mujeres y hombres y del silencio y la palabra tomando como metáfora el tándem Eco-Narciso. En el caso de Hollander, y partiendo ya desde la recepción de la ninfa en la literatura occidental, el autor plantea cinco perspectivas fundamentales que articulan la estructura de su libro: "Echo Acoustical", "Echo Allegorical", "Echo Schematic", "Echo Metaphorical" y "Echo Metaleptic". La investigación de Hollander es de extrema importancia, pues cubre un vacío considerable hasta el momento no sólo en la mitografía sino también en los estudios de la crítica literaria. Si bien el primero de los capítulos ahonda más en la cuestión del estudio del mito desde la perspectiva del doble fónico, de los cinco espacios trabajados por el autor es necesario destacar el capítulo dedicado a la metalepsis, pues profundiza en los ecos de la intertextualidad, fundamentales en los estudios de recepción, así como la aproximación que realiza a los orígenes formales y mitológicos de Eco como tropo figurativo. Esta última permite a Hollander abordar su objeto de estudio también desde el análisis retórico.

Como discípulo de Hollander, el trabajo de J oseph Loewenstein Reponsive Readings: Versions of Echo in Pastoral, Epic, and the J onsonian (1984) supone la continuación de The Figure of Echo y presenta el fenómeno del eco como topos de la literatura pastoral, la introducción de la ninfa en la imaginación mitopoética y, finalmente, su presencia en el teatro del Renacimiento británico también en calidad de doble discursivo (Loewenstein 
1984: 2). Eco es estudiada como continuación del fenómeno natural que lleva su nombre, como corporeidad-personificación y repetición-resonancia, desarrollada en la obra dramática y poética de George Gascoigne, Ben J onson o Milton, por ejemplo.

En la línea feminista de Mizzau, y coincidiendo con la segunda edición de su ensayo Eco e Narciso, en 1988 Naomi Segal publica Narcissus and Echo: Women and the French Récit, obra en la que emplea ambas figuras como metáfora central de los argumentos del género confesional francés récit que Segal define como sigue:

In the French genre of the confessional récit, the protagonist is a young man, mismothered, unable to love; he recounts the story of his failure to a paternal interlocutor and frame-narrator who serves him as double and invites him, through confession, to enter the patriarchal order. The stake is a female character who, in almost every case, dies (more or less by his wish), releasing him to tell the tale. Her absence is necessary for him to narrate. But, as Gide's Michel puts it, 'at times I am afraid that what I have suppressed will take revenge.' There remains in each of these texts the trace of the woman's voice, uttering her desire and her knowledge, to haunt the male couple. (1989: 169-170)

En trabajos posteriores, Segal completa sus interpretaciones del mito siguiendo la línea trazada por la crítica feminista al insistir en "getting the woman's voice out of the man's mirror" (2000:141). La duplicidad que trabaja la autora y que bañará recreaciones contemporáneas de la ninfa ovidiana está basada en la dicotomía hombre-mujer/ palabrasilencio que será retomada en ensayos posteriores como el de Luisa Campuzano Eco y Narciso (2006) acerca de la literatura latinoamericana escrita por mujeres.

En 1994, un año después de la publicación del magnífico artículo de Spivak 'Echo', Joseph Pivato explora en Echo. Essays on other Literatures lo que el autor denomina literatura de minorías', es decir, "texts that are marginal to the literary canons in Canada", (2003:39) en una Canadá que ya supone para la tradición eurocentrista una periferia. Pivato, inmigrante italiano asentado en Canadá, inicia el primer capítulo de su estudio de este modo:

Echo: the sound that is reflected back as sound waves of a first or previous sound. The echo answers back the first call as an imitation. In a sense, literary criticism is a reflection of, and a reflection on, the primary texts produced by the creative writers. It is a response to the initial call. Echo comes from the Greek, echo. In French it is echo; in Italian eco.

Echo can also be a homonym for ecco, the Italian word for behold. Eccomi, here I am in answer to a call, if not a calling (I Samuel, 3). The call comes from the works of ItalianCanadian writers and other ethnic minority writers in Canada. These first texts cannot be ignored; they call out to me and to other readers. These new voices call out to us asking to be heard (2003:29).

La duplicidad de la identidad y el discurso que escucha Pivato en esta literatura de minorías representa la tensión entre poder/contrapoder que ya había sido perfilada por Gayatri Spivak en 1993 con 'Echo'. En este artículo, Spivak realiza una revisión del 
concepto narcisismo —en su herencia psicoanalítica que considera eurocentristarescatando la figura de Tiresias. Para Spivak, una oposición fundamental olvidada generalmente por la crítica sitúa a Eco y a Tiresias, en tanto que figuras castigadas por la acción divina, en los extremos del episodio de las Metamorfosis en los que se detalla la historia de la ninfa. Si recordamos, en Met.III, 323-339 Ovidio relata la historia de cómo Júpiter suaviza el castigo de J uno a Tiresias ofreciéndole el don de la visión futura; cosa que no sucede en el episodio de Eco. El hecho de enmarcar la historia de Narciso entre la experiencia asimétrica del castigo de Tiresias y Eco es el motor que inicia la lectura de Spivak fundamentada en las siguientes premisas:

The story of Narcissus is a tale of the construction of the self as an object of knowledge. I will suggest below that the account of Echo is a story of punishment that is finally a dubious reward quite outside the borders of the self. The story of Narcissus is framed, then, in the value-coding or gendering of affect in a spectacular dynamics of transgression and reward (1993: 23).

Desde este espacio vacío de la recompensa del sujeto femenino que ocupa Eco, Spivak se aproxima a la ninfa a partir de las posibilidades de deconstrucción del mito desde el concepto de la otredad y el espacio de la respuesta.

La inclusión del episodio de Ovidio como metáfora de la relación entre culturas dominantes y dominadas a través del arte es retomado por Ricky Burnett en su trabajo South African Art: A Story of Echo, Narcissus and Blind Tiresias (1997). ${ }^{1}$ El centro de los argumentos de Burnett, en su cuestionamiento de los objetos y espacios de representación del arte sudafricano, quiere dar respuesta a la siguiente cuestión que plantea al comienzo de cada uno de los dos ensayos recogidos en el volumen:

Our challenge and the point of my polemic is to ask which world should now be with us as we move to a new millennium under a new flag with a new constitution? (...) where will we find the social body within which Echo can re-find her voice and within which Narcissus can find a true and substantial insight into a meaningful other? (1997: 4).

In today's presentation Echo and Narcissus are going to take the stage. They, I hope, are going to help dramatise the operation of two numbing, even deadening, attributes of advancedness or are associated with the championing of advancedness in our country. They stand for a poverty of spirit which occurs when thoughts cannot be reframed unless in previously established terms; when discourse supplants experience as the primary ground of image-making; when 'place' becomes a floating mental operation; and, when interiority and its uncertainties supplant exteriority and its assertions (1997: 1-2).

\footnotetext{
${ }^{1}$ La publicación es el resultado de las conferencias ofrecidas por Ricky Burnett inscritas en el evento Scotland Africa '97 organizado por el Centre of African Studies de la University of Edinburgh coincidiendo con la exposición de J ackson Hlungwani Image and Form en el College of Art de Edimburgo.
} 
En la línea del estudio de género de Segal, y con la oposición este/ oeste, norte/ sur que plantea Burnett, descubrimos además en Eco la palabra que también Luisa Campuzano, como hemos mencionado con anterioridad, esboza como epítome de las rescrituras de mujeres latinoamericanas de los mitos de occidente, y que será una de las lecturas más habituales que copen la historia sígnica de la ninfa a partir de las últimas décadas del siglo XX. ${ }^{2}$ Ya en 1979, por ejemplo, Pivato apuntaba en el capítulo 'Voices of Women' de su estudio, paralelismos claros entre la experiencia de silenciación de Eco y la de las escritoras de origen italiano en Canadá que "have had to look into their history to create a female reality and to give voice to that reality" (2003: 129-130). Eco se viste, pues, como la palabra femenina que se enfrenta a la creación lingüística patriarcal, al tiempo que la dicotomía centro/ periferia que suscita su relación con Narciso la convierte en metáfora de la escritura del imperio contra la metrópoli. ${ }^{3}$

Volviendo a 1997, a la vez que la monografía de Burnett, Patrick O’Donell publica Echo Chambers: Figuring Voice in Modern Narrative (1997). Los mitos de Narciso y Eco sirven al autor como pretexto del eje central de su argumento: el estudio de la representación de la voz y la construcción de la identidad en la narrativa moderna a partir de los textos de Charles Dickens Our Mutual Friend, Ulysses de James Joyce, Absalom, Absalom! de William Faulkner, Under the Volcano de Malcom Lowry y JR de William Gaddis. Con un objetivo similar pero desde diferentes disciplinas publican en 1991 y 2005 respectivamente Amy Lawrence Echo and Narcissus: Women's Voices in Classical Hollywood Cinema y Peter Doyle Echo and Reverb: Fabricating Space in Popular Music, 1900-1960. El motivo del doble en estos casos se fundamenta en la ausencia/presencia, en el estudio de O’Donell; de nuevo la dicotomía hombre-mujer/palabra-silencio en Lawrence y en el fenómeno de la repetición acústica en el trabajo de Doyle.

En 1998 Antonio Ballesteros regresa al estudio de la recreación del mito de Narciso (y por ende el de Eco) en la narrativa, esta vez victoriana y con mayor éxito que sus antecesores, en Narciso y el doble en la literatura fantástica victoriana (1998). Si bien de nuevo el motivo del doble en Eco se encuentra supeditado una vez más a la figura de Narciso, es destacable de la obra de Ballesteros su análisis de la presencia de ambos mitos en sus fuentes clásicas, así como su acercamiento a las diferentes interpretaciones que ha suscitado la figura de Narciso, y como consecuencia la de Eco, en las letras inglesas.

Finalmente, ya en el siglo XXI, destacamos tres trabajos que continúan la tradición iniciada por sus antecesores completando las diferentes lecturas del mito que han sido trabajadas con mayor intensidad en la literatura occidental. En 2003, Maurizio Bettini y Ezio Pellizer dedican el volumen Il mito di Narciso. Immagini e racconti dalla Grecia a oggi a un estudio de la figura de Narciso desde sus fuentes clásicas hasta la actualidad. Si bien no existe en el libro ningún capítulo específico dedicado a la relación entre el motivo

\footnotetext{
2 Véanse, por ejemplo, Dobrian (1995); Fischman (1992); Raoul (1990).

3 Sobre esta cuestión véanse Naik (1980-1) y Levy (2002).
} 
del doble y refiguraciones modernas de Eco, destacamos la utilidad del volumen para el estudio de la relación con su contrapunto ovidiano por la amplitud cronológica del mismo.

En el año 2000 salen a la luz los últimos dos trabajos que deseamos comentar en estas páginas: la colección de ensayos editada por Lieve Spaas y Trista Selous Echoes of Narcissus, ${ }^{4}$ y la monografía de Veronique Gély-Ghedira La nostalgie du moi: Écho dans la littérature européenne acerca de la pervivencia de Eco en la literatura moderna. En el primero de estos trabajos a pesar de las constantes referencias a la ninfa, es de destacar únicamente el capítulo de Segal para el estudio de Eco y el doble en la literatura. Segal retoma sus argumentos expuestos a finales de los ochenta e insiste en la duplicidad Eco/Narciso, y no tanto en el sentido de extraer la voz femenina del espejo masculino, sino como "masculinity that seeks to define itself against femininity" (2000:6). La nostalgie du moi: Écho dans la littérature européenne de Gély-Ghedira es tal vez el más completo trabajo dedicado a la figura de Eco de los que tratamos en estas páginas desde Hollander en 1981. En su recorrido desde la Edad Media hasta el siglo XX, la autora enriquece las aportaciones realizadas acerca de la recepción de la ninfa con ejemplos abundantes de la duplicidad intrínseca al mito desde el punto de vista de la poética, la retórica y la identidad, por ejemplo.

De esta selección no exhaustiva de monografías dedicadas a la presencia de Eco en la literatura occidental podemos concluir cómo el desdoblamiento de la ninfa en Ovidio predomina frente a otras versiones latinas del mito que la unen a Pan, por ejemplo, en Longo. ${ }^{5}$ De su contraposición a la figura de Narciso en Ovidio, como hemos presentado hasta el momento, confluyen diversas formas de duplicidad a partir de dos ejes: la naturaleza intrínseca del mito y la oposición de éste frente a la figura de Narciso. En el primero de los casos nos hallamos ante cuestiones como el doble fónico o el cartesiano; en el segundo, entran en la ecuación otros aspectos culturales que transforman la dicotomía en la oposición de pares como hombre/mujer, masculino/femenino, metrópoli/colonia, centro/periferia que estructuran el funcionamiento de las sociedades contemporáneas. Tras este breve recorrido por los estudios acerca de la pervivencia de Eco en el mundo occidental, el siguiente apartado de nuestro trabajo aborda algunas destacadas revisiones del mito en las últimas décadas en la literatura escrita en lengua inglesa como preludio al análisis de la presencia del mito en el poemario temprano de Moniza Alvi How the Stone Found Its Voice (2005).

\footnotetext{
4 Este volumen se concibe a partir del "International Colloquium” celebrado en el Institut Français de Londres en Mayo de 1997.

${ }^{5}$ Véase por ejemplo el listado de obras que Reid (1993) incluye bajo la entrada de Narciso.
} 


\section{RECREACIONES CONTEMPORÁNEAS DE ECO EN LA LITERATURA EN}

\section{LENGUA INGLESA}

Los trabajos revisados en el anterior apartado de nuestro estudio muestran los perfiles de la figura de Eco que han copado las rescrituras del mito en las últimas décadas del siglo XX y los inicios del XXI. En las siguientes páginas proponemos un recorrido sucinto por algunas de estas rescrituras que condensan las lecturas planteadas desde Colby en 1920 hasta Bettini y Pellizer en 2003, prestando especial atención al poemario How the Stone Found its Voice (2005) de Moniza Alvi.

La primera revisión del mito de Eco con la que iniciamos nuestro recorrido data de 1935 y viene de mano de uno de los exponentes de la literatura en lengua inglesa del siglo XX, Samuel Beckett. En 1935 Samuel Beckett publica el poemario de juventud Echo's Bones con el mismo título que el relato Echo's Bones and Other Precipitates, y concebido en sus inicios como el desenlace de More Pricks than Kicks, publicada en 1934. Varias percepciones del doble literario se dan cita en el conjunto de trece poemas que forman la colección: la presencia/ausencia del individuo; el silencio frente a la palabra/ escritura; la tensión entre construcción/deconstrucción o la dicotomía mente/cuerpo, por ejemplo. El eje argumental del poemario es la descomposición infinita del universo frente a la impertérrita mirada del yo-lírico, su autor, que participa del mismo deterioro. Los huesos de Eco que promulga en su título se convierten en los huesos del mundo, los del individuo y los de la palabra que a su vez se transforman en piedra, en nada. El resultado, una nueva dualidad: destrucción y construcción. Como señala Talens, "Nada hay más constructivo que la destrucción, (...) Destruir las palabras, agotar su caudal, mientras lo haya. Ése es el papel del escritor" (1979: 31). La idea, que el autor plantea desde el primer poema, The Vulture', quedará grabada en el conjunto de su producción literaria:

dragging his hunger through the sky

of my skull shell of sky and earth

strooping to the prone who must

soon take up their life and walk

mocked by a tissue that may not serve

till hunger earth and sky be offal

Entre la infinitud de interpretaciones que suscita la Eco de Beckett hallamos la eco metafórica de Hollander que abre sus puertas, a partir de la (no)representación de su doble, a la deconstrucción postestructuralista y a crisis del individuo que baña el siglo XX.

En 1989, Marlene Nourbese Philip publica la colección de poemas She Tries Her Tongue, Her Silence Softly Breaks. Estructurada en una introducción y nueve poemas, la voz poética de Philip representa a una Eco contestataria e imaginativa que desdobla el discurso de Narciso hasta tropezar con la fórmula que la transforme en sujeto agente de la 
enunciación. Philip procede de la tradición de escritura femenina afro-caribeña asentada en Canadá. Como tal, y al igual que autoras de diferentes tradiciones como las irlandesas Eavan Boland o Nuala Ní Dhomhanaill, la pluma de Philip es la Eco sobre la que teorizan Spivak (1993), Pivato (1994), Burnett (1997) y Campuzano (2006). Es la voz de mujer que desde las antiguas colonias desafía a una metrópoli patriarcal que silencia y domina su discurso. La propia Philip plantea la cuestión con estas palabras: "the relationship between Prospero and Caliban has been a blueprint for the problematic that language presents for colonial peoples who trace their linguistic genealogy back to Caliban" (1997: 165). Los dobles de Eco que se plantean, pues, en el poemario de la autora parten de la teoría feminista y postcolonial ahondando fundamentalmente en cuestiones acerca de la identidad del individuo y la construcción del lenguaje.

Marlene Nourbese Philip nació en Tobago y tras sus estudios en Economía en The University of the West Indies, una licenciatura en derecho en la University of Western Ontario y un Máster en ciencias políticas dedicó los primeros años de su vida laboral al ejercicio del derecho hasta 1993, cuando decide dedicarse plenamente a la escritura. Su producción literaria es eminentemente poética. El trabajo que aquí comentamos, publicado en 1989, fue galardonado en su manuscrito con el premio Casa de las Américas. De los nueve poemas del volumen, tan sólo 'And Over Every Land and Sea' se dedica explícitamente a la revisión de la mitología clásica, en concreto, al mito de Prosérpina. Sin embargo, el conjunto del poemario es un intento de recuperar la voz petrificada de Eco, de un individuo aniquilado por el poder de la metrópoli y condenado a repetir el discurso del siempre hegemónico Narciso. Así lo exponen los poemas 'Discourse on the Logic of Language' y 'Universal Grammar', reflexión en torno al par silencio/ palabra o 'Meditations on the Declension of Beauty by the Girl with the Flying Cheeckbones', acerca de la 'de'petrificación del sujeto abatido por su condición de otro:

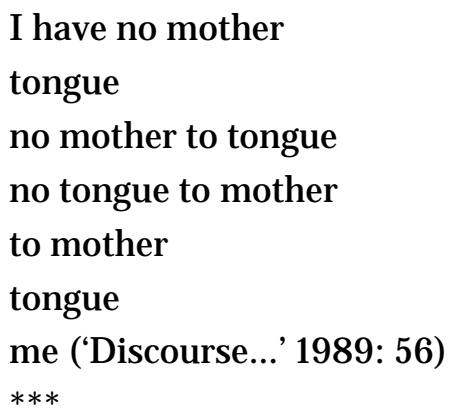


En 1988 Eco también aparece en los escenarios británicos como personaje secundario de The Love of the Nightingale de Timberlake Wertenbaker. Nacida en el País Vasco francés, Wertenbaker combina la sátira con la visión utópica para recrear en sus textos el discurso de voces silenciadas por problemáticas contemporáneas. En The Love of the Nightingale, puesta por primera vez en escena el 28 de octubre de 1988 en The Other Place, de Stratford-upon-Avon, de manos de la Royal Shakespeare Company, Wertenbaker recrea el episodio ovidiano de Procne y Filomela. Sus fuentes clásicas son varias: las Metamorfosis de Ovidio (IV. 424-674), el Hipólito y las Bacantes de Eurípides y el Tereo de Sófocles. En Wertenbaker, Eco forma parte - junto a Hero, Iris, J uno y Helena- de un coro femenino que, como contrapunto del coro masculino, da voz al silencio de las dos hermanas tras los abusos del implacable Tereo. En sus palabras, la Eco de Wertenbaker recuerda el análisis de Mizzau (1979) y Segal (1988), o posteriormente Pivato (1994), que socavan las relaciones de poder y de género en la tensión entre Eco y Narciso. Cuando las palabras de Eco se individualizan y escapan de la homogeneidad del coro se fragmenta el hilo discursivo a favor de la construcción nominal. Esta ruptura lingüística también presente en Beckett es en esta obra signo característico de la subjetividad de la ninfa como demuestran los siguientes ejemplos:

Echo: Gone, Procne, the words? ...

Echo: Silent, Procne, who? ....

Echo: Tereus....

Echo: Tereus....

Echo: A beating of wings... (2000: 30)

Echo: Tereus....

Echo: A welcome.......

Echo: Silent. (2000: 43)

Echo: Image, Echo....

Echo: No end....

Echo: Metamorphosis. (2000: 67)

Un segundo rasgo que evoca en Wertenbaker a la Eco ovidiana es la marca de la repetición, aunque en este caso como apropiación del espacio del agente del discurso que representa a lo largo de toda la obra. Como demuestra el ejemplo que ofrecemos a continuación, Eco reformula el significante de la imagen/metáfora ampliando su significado al incorporar un abanico de referencias que pertenecen a su propia experiencia. 'Image', 'Echo', 'Metamorphoses' son todas parte del campo semántico del discurso que explicita los esfuerzos del personaje por liberarse de las cargas impuestas por su doble Narciso (el discurso patriarcal/ la metrópoli...):

Hero: The sky was so dark this morning...

Procne: It'll rain. It always rains.

Iris: Again. 
Hero: I was not talking meteorologically. Images require sympathy.

Echo: Another way of listening. (2000: 43)

J une: We show you a myth.

Echo: Image. Echo. (2000: 64)

En 1997 Eco reaparece en las páginas de Tales from Ovid, revisión poética del afamado Ted Hughes de las metamorfosis de Ovidio. El éxito del poemario hace que tan sólo dos años más tarde, en 1999, Tim Supple y Simon Reade realicen una versión dramática del mismo que se estrena en 1999 en el Swan Theatre de la Royal Shakespeare Company. La Eco de Hughes, y por ende la de Supple y Reade, bebe del animalario del poeta laureado al indagar en los vínculos más desgarradores entre el ser humano y una naturaleza sensorial y primigenia. La dualidad del mito latente en la revisión de Hughes es la que Mizzau plantea en su trabajo de 1979 llevada al extremo de la pasión descontrolada no correspondida. Así, y como parte de un macrocosmos superior que rige el ciclo vital de las bestias que lo habitan, la Eco de Hughes es una joven ardiente y risueña que perpetúa roles femeninos contemporáneos a merced de la voluntad de su doble. Supple y Reade despojan a Eco de estos patrones modernos y centran su revisión del texto en la tensión Eco/Narciso:

Narcissus: Where are you?

I'm here.

Echo: [catching at the syllables as if they are precious]

I'm here, I'm here, I'm here, I'm here.

Narcissus: (looking around wildly)

I'll stay here.

You come to me.

Echo:

Come to me,

To me, to me, to me.

[Narcissus stands baffled

whether to stay or go.

He begins to run.]

Narcissus:

Stay there.

Echo: (weeping)

Stay there,

Stay there, stay there, stay there.

[Narcissus stops and listens.]

Narcissus [more quietly]

Let's meet halfway. Come.

Echo: (eagerly)

Come come come come. 
[Echo emerges from the underground,

her expression pleading,

her arms raised to embrace him-

Narcissus turns and runs.]

Narcissus:

No, no, I would sooner be dead

Than let you touch me.

Echo: [collapsing in sobs, voice lurching]

Touch me, touch me, touch me, touch me.

(Tiresias):

Echo moped under the leaves.

Humiliated, she hid

In the deep woods (1999: 14-15)

Ya en el siglo XXI Philip Terry edita Ovid Metamorphosed, publicado en la editorial Vintage en el año 2001. La colección de Terry reúne a plumas de renombre de la tradición en lengua inglesa en un total de diecinueve relatos cortos que no hacen sino constatar la creciente intensidad con la que la producción ovidiana está presente en la literatura inglesa contemporánea. La aportación de Nicole Ward J ouve al volumen es Narcissus and Echo', relato en el que la autora reflexiona sobre las relaciones humanas contemporáneas a partir del reflejo de la identidad y el doble de la voz. A partir del microcosmos de un congreso académico organizado a propósito de la producción literaria de la escritora Miranda, Jouve presenta al lector un amplio abanico de personajes que cimentan su identidad en la tensión uno/ otro concebida desde el epicentro, Narciso/Miranda, desde donde irradian todos sus dobles:

Andy started. Stopped and started again. A quotation. Then another. Quotations not even strung together. He was a translator, Heloise reminded herself. Perhaps if you were a translator you were especially dependent on other people's words? When called upon to say his own thing, all Andy could do was echo other people's words. They had a strange meaning in his mouth. He was looking at Miranda, heroically seated in her customary place in the front row, exhibiting again, Heloise thought, her admirable self-control (2001: 127).

Despojado de la perspectiva postcolonial y de género de Pivato o Campuzano, por ejemplo, los dobles de J ouve son los dobles de Eco estudiados por éstos a partir de las relaciones de poder entre individuos.

En 2003, A.S. Byatt, gran novelista e impulsora de la ficción neovictoriana en la tradición británica, publica The Little Black Book of Stories, una colección de también cinco relatos breves en los que se encuentra The Stone Woman'. 6 Basado en su experiencia personal tras una larga estancia hospitalaria, Byatt retoma mitos ovidianos de petrificación

\footnotetext{
${ }^{6}$ A.S. Byatt contribuyó al volumen editado por Philip Terry con 'Arachne'. Véase Terry (2000: 131-157).
} 
femenina para reflexionar acerca de la descomposición y materialidad del individuo y la escisión cartesiana mente/ cuerpo. No existe una referencia directa a la ninfa pero sí una búsqueda persistente de su presencia en la (de)construcción psicológica y social del cuerpo femenino y espacios comunes de la vida contemporánea:

Slowly, slowly, day by quick day, her torso was wrapped in a stony encrustation, like a corselet. She could feel that under the stones her compressed inwards were still fluid and soft, responsive to pain and pressure. (2003:139)

Whether or not she became wholly inanimate, she must find a place to stand in the weather before she became immobile. She visited city squares, and stood experimentally by the rims of fountains, or in the entrances of grottoes [...] What she saw was a flat stony city, house after house under the humped ripples of earth, marked by flat stones, standing stones, canted stones, fallen stones, soot-stained, dropping-stained, scum-stained, crumbled, carved, repeating, repeating. She walked along its silent pathways, past dripping yews and leafless birches and speckled laurels, looking for stone women. They stood there- or occasionally lay fallen there - on the rich earth (2003:148-9)

Desde Samuel Beckett hasta A.S. Byatt el recorrido por la pervivencia de la figura de Eco que hemos ofrecido en estas páginas no es sino una reducida muestra del potencial literario de la ninfa. Si tan solo consideramos traducciones, versiones o refiguraciones del mito en lengua inglesa, la figura de Eco ha sobrevivido con menor intensidad al paso del tiempo en comparación con otras homólogas ovidianas. ${ }^{7}$ Sin embargo, si en nuestra aproximación al mito contemplamos las diferentes formas de mirar la continuidad de la literatura clásica en la cultura moderna que plantea Hardwick en su conocido trabajo Reception Studies - 'Acculturation', 'Adaptation', 'Analogue', 'Appropriation', 'Authentic', 'Correspondences', 'Dialogue', 'Equivalent', 'Foreignization', 'Hybrid', 'Intervention', 'Migration', 'Refiguration', 'Translation', 'Transplant' y 'Version' (2003: 9-10) - la presencia de Eco en la tradición británica se multiplica de forma exponencial hasta llegar a ser inconmensurable. En nuestra opinión, el motivo de este incremento radica precisamente en la naturaleza dual del mito en Ovidio que, desde el castigo de J uno hasta el reflejo de su doble discursivo, Narciso, es estandarte de oposiciones binarias. En el siguiente y último apartado de nuestro estudio rescatamos una reciente colección de poemas que bajo el título How the Stone Found its Voice (2005) ilustra algunas de las tendencias de revisión del mito que bajo diferentes concepciones del motivo del doble han copado el último siglo.

\footnotetext{
${ }^{7}$ Véase por ejemplo Marshall (1998) acerca de recreaciones modernas de los mitos de Pigmalión y Galatea.
} 


\section{LOS DOBLES DE ECO: HOW THE STONE FOUND ITS VOICE (2005) DE MONIZA ALVI}

Moniza Alvi nació en Lahore, Pakistán, en 1954, aunque poco tiempo después se trasladó a Hertfordshire, Inglaterra, país del que haría su residencia hasta hoy. Fue educada en las universidades de York y de Londres e inició su carrera en la enseñanza hasta que en 1991 fue galardonada con el Poetry Business Prize con Peacock Luggage, colección de poemas escrita junto a Peter Daniels (Ray 2007: 9). Tras ésta, sus dos primeros libros de poesía, The Country at My Shoulder (1993) y A Bowl of Warm Air (1996) fueron publicados por Oxford University Press. Su trabajo ha sido estudiado junto al de otras escritoras en lengua inglesa de origen asiático como Meera Syal y Sujata Bhatt heredando también tintes del lirismo de Mimi Khalvati (Dowson y Entwistle 2005: 203; Sage 1999: 14). Según Bassnett, la escritura de Alvi deriva de:

her experience as a woman belonging to more than one culture, and that hybridity is an essential part of her creativity. She writes from her own experience as being in-between, a woman with a place in two cultures and consequently perhaps not entirely located in either. Her writing is therefore emblematic of the position occupied by millions in today's world. A theme to which she returns in many poems is that of belonging and not-belonging (1998: 61).

La propia Alvi se refiere al hibridismo de su obra señalando la importancia de los dos polos de la identidad dual que se refleja en su escritura: "the poems that do not concern my Asian background are equally important to me.. I have written about Pakistan partly because it was, in the first instance, fantasy" (Sage 1999: 14-15). La fusión de esta aparente dicotomía a partir de la recuperación del discurso es el punto de partida de nuestro análisis de How the Stone Found its Voice.

How the Stone Found its Voice es una colección de poemas estructurada en seis secciones principales y publicada en Bloodaxe en 2005. El hilo argumental del volumen es, según Bainbridge, "[an] overriding sense of not belonging, of fragility, even in our relationship with the self" (2005). Este sentido de no-pertenencia se acentúa en los dos primeros bloques del poemario que abordan, respectivamente, la potencial dualidad intrínseca a la experiencia de la creación y a la vida cotidiana del sujeto contemporáneo.

La primera parte del volumen es de la que toma su título con el último poema de la misma, 'How the Stone Found its Voice'. Con el motivo de 'How...' Alvi realiza un recorrido por diferentes mitos de creación occidentales haciendo hincapié en la (de)construcción de la vida y la escisión de la materia. ${ }^{8}$ El mundo, los países, las ciudades, los animales, e

\footnotetext{
8 Los títulos de los poemas que forman esta parte son los siguientes: 'How the World Split in Two', 'How the Countries Splipped Away', 'How the Answers Got Their Questions', 'How the City Lost Its Colour', 'How the Animals Tried on Our Clothes', 'How the Sky Got a Hole in It', 'How the Words Feared the Mouth', 'How the Long Way Off Rolled Itself Up', 'How the Children Were Born', 'How Thought Accompanied the Traveller', 'How Yesterday J oined Today and Tomorrow', 'How the Stone Found Its Voice'.
} 
incluso las preguntas y respuestas están compuestos de mitades irreconciliables que, como Eco, soportan la certeza de la imposibilidad de la armonía entre sus partes. Así lo expresa Alvi en 'How the World Split in Two' o 'How the Countries Slipped Away'. En la primera de estas composiciones la autora ahonda en una desmembración atroz del planeta que desde 'How the Countries...' y en poemas sucesivos de la sección se traslada a una desintegración cultural y del individuo:

\section{Was it widthways or lengthways, a quarrel with the equator? \\ Did the rawness of the inside sparkle?}

Only this is true:

there was an arm on the one side and a hand on the other, a thought on one side and a hush on the other.

\section{And a luminous tear} carried on the back of a beetle went backwards and forwards from one side to the other (2005: 9)

De esta sección dos poemas son destacables en el estudio de la duplicidad de la identidad, del silencio y la palabra de Eco: 'How the Word Feared the Mouth' y 'How the Stone Found its Voice'. En el primero de ellos, tras haber profundizado en los inicios de la creación en los seis poemas que abren esta primera parte —ciudades, países, animales-, Alvi introduce la cuestión del discurso, la palabra. Sin embargo, lejos de la antropomorfización de la misma en una entidad superior, como sucede en la tradición judeocristiana, Alvi excava en los cimientos del nacimiento de ésta en su oposición a un estado anterior de calma, de silencio, de existencia en un limbo que no deja al lenguaje al abandono del abismo de los labios, la boca, la hendidura por la que caerán para siempre:

Some words lay quietly,

they didn't wish to be roused,

while others were restless,

waiting for the drama to begin.

which could fill with potatoes and beer.

Half-formed words were often lost.

And others were equally unfortunate-

it was their fate

to be cruelly nailed to the air. (2005: 15$)$ 
Si consideramos como base la Eco metafórica de Hollander, la ninfa ovidiana se encuentra aquí presente del mismo modo que en 'Meditations on the Declension of Beauty by the Girl with the Flying Cheek-Bones' o 'Discourse on the Logic of Language' de Marlene Nourbese Philip, en los que la autora proponía el balbuceo, la dificultad de la palabra desde una lengua patriarcal impuesta por una metrópoli. Si bien el sentido de otredad latente en la poesía de Philip, y que recogen los trabajos de Campuzano (2006) o Pivato (1994) no es tan evidente en esta composición, sí perdura la dicotomía palabra/ silencio dentro de la tensión que mantiene viva la pervivencia de Eco.

En la segunda de las composiciones aludidas más arriba, 'How the Stone Found Its Voice', que cierra la primera sección del poemario, la dificultad de enunciación convive con una postura más política. El sujeto agente del discurso es 'the stone', que puede evocar tanto una entidad mágica o divina en culturas primigenias, como cualquier sujeto petrificado por motivos culturales, políticos o de género en la sociedad occidental. Tras un doloroso alumbramiento nace la palabra. Una palabra firme y rebelde que insta a la acción y condena la apatía:

We had waited through so many lifetimes

for the stone to speak, wondered if

it would make compelling pronouncements, anything worth writing down.

Then, after the war of wars

had ground to a shattering halt, the stone

emitted a small grinding sound rather like

the clearing of a throat.

Let us be indifferent to indifference,

The stone said.

And then the world spoke. (2005:20)

La búsqueda de espacios fronterizos entre estructuras binarias pasa en la segunda sección del poemario a la dicotomía este/oeste, colonia/metrópoli, identidad/otredad. Como afirman Dowson y Entwistle, Alvi "sees writing as 'an act of discovery on the border where inner and outer worlds meet; exploring her own cultural complexity creates something positive about what was, in childhood and adolescence, rather unsettling and difficult to think about" (2005: 203). Un total de once poemas encabezados por 'Half-andHalf' recorren objetos y escenas de la vida cotidiana de Alvi en Inglaterra que abren puertas hacia la (de)construcción de la identidad del sujeto británico de origen pakistaní desde la amenaza del otro. Así se muestra en 'The Suits' o 'Rural Scene', en los que 
imágenes frecuentes de la vida común inglesa se yuxtaponen con estampas inquietantes y ajenas a la ortodoxia cultural del establishment:

My father's forties suit, bought when he first came to England,

pin-stripped with broad lapels, comfortingly chocolate, but crisp.

He and his Pakistani friends and their we-have-arrived-suits

$[\ldots]$

his suit. He's handsome as a doctor, our neighbour said

$[\ldots]$

This was before Go back home! Their suits of armour

could have stood up without them. Walked on and on. ('The Suits' 2005: 23)

$* * *$

The luminous Norfolk skies,

the tractors, the gunshots,

the still ponds, the darting rabbits,

cow parsley by the field gates-

all are re-imagining themselves

because Tariq walks in his village,

part of the scene, yet conspicuous,

as if he is walking a tiger. ('Rural Scene' 2005: 29)

La escisión cultural del mundo exterior es también geográfica y se prolonga hacia el individuo en 'Half-and-Half' y en 'England, I Am Gazing at Your Body', donde la dimensión material de la dualidad de Eco se traduce en una lucha del individuo contra su propia fisionomía y la de los dos países, las dos culturas que le dan identidad:

The thin line running from my navel downwards

meant, I thought, that I was half-and-half,

like the coffee my mother drank in restaurants.

That was sophisticated-

but to be half-and-half oneself? And one part

stemmed from a country that wasn't whole- ('Half-and-Half' 2005:2)

$* * *$

all I can do is sniff you, your industrial belts,

on my mercy, you ask for a grain

of understanding-

all I can do is sniff you, your industrial belts

$[\ldots]$ 
England, it's time

to call the children in.

I pull myself off you.

Hard to prise my stickiness from yours. ('England.. 2005: 28)

Únicamente una composición en las dos secciones analizadas en este trabajo propone una posibilidad de reconciliación de los dos fragmentos que conforman la unidad del sujeto estudiado en Alvi. Cerrando el segundo bloque de poemas trabajados, 'For my Daughter' insiste en la escisión física, mental y cultural del individuo aunque abre puertas al restablecimiento de la unidad de nuevo por medio de espacios y procesos naturales que constituyen el punto de encuentro entre las partes:

Your name rests for a moment on my tongue

and speaks resoundingly of Englishness

I let it stay a little longer

and it swells and fluctuates.

You are here with a whisper

of another continent in your bones,

though you can't yet think of it like that.

And perhaps we are all immigrants

in these towns and villages,

and all strangers to ourselves.

When I say your name it melts like ice,

or starts to grow-

a hospital, a playground,

a path through the fields, the sky (2005: 33)

Si consideramos el poemario de Alvi dentro del conjunto de obras expuestas en este trabajo, podemos concluir que la que aquí interpretamos como su recuperación particular del mito de Eco no es sino resultado de la evolución del concepto del doble en el pensamiento occidental. De Beckett a Alvi, el potencial del mito es manifiesto en la literatura en lengua inglesa como portador de esta condición sine qua non. De mero recurso retórico, Eco viaja por reflejos de identidades y oposición de culturas. Por el anverso y el reverso de la (in)comunicación del sujeto moderno. 


\section{BIBLIOGRAFÍA}

Bainbridge, Charles (2005). "Strangers to Ourselves", The Guardian (17.12.2005) $<\mathrm{http}$ // / books.guardian.co.uk/ review/ story/ 0,12084,1668483,00.html>(26.9.2007).

Ballesteros, Antonio (1998). Narciso y el doble en la literatura fantástica victoriana. Cuenca: Ediciones Universidad de Castilla-La Mancha.

Bassnett, Susan (1998). "Transplanting the Seed: Poetry and Translation" en Susan Bassnett y André Lefevere (eds.), Constructing Cultures: Essays on Literary Translation. Clevedon: Multilingual Matters: 57-75.

Bettini, Maurizio y Pellizer, Ezio (2003). Il mito di Narciso. Immagini e racconti dalla Grecia a oggi. Torino: G. Einaudi.

Burnett, Ricky (1997). South African Art: A Story of Echo, Narcissus and Blind Tiresias. Edinburgh: University of Edinburgh.

Campuzano, Luisa (2006). Eco y Narciso. Buenos Aires: Editorial La Bohemia.

Colby, Eldridge (1920). The Echo Device in Literature. New York: The New York Public Library.

Dobrian, Susan Lucas (1995). "Echo's Revenge in Three Spanish Narratives by Women". Letras Peninsulares 8, 1: 169-79.

Dowson, Jane y Alice Entwistle (eds.), (2005). A History of Twentieth-century British Women's Poetry. Cambridge: Cambridge University Press.

Fischman, Susan (1992). "Even As We Speak: Womens Voice and the Myth of Echo" Dissertation Abstracts International 52, 12: 4321A-22A.

Gély-Ghedira, Veronique (2000). La nostalgie du moi: Écho dans la littérature européenne. Paris: PUF.

Hardwick, Lorna (2003). Reception Studies. Oxford: Oxford University Press.

Hollander, J ohn (1981). The Figure of Echo. A Mode of Allusion from Milton and After. Berkeley, Los Angeles, London: University of California Press.

Hughes, Ted (1999). Ted Hughes' Tales from Ovid. Adapted by Tim Supple and Simon Reade. London: Faber \& Faber.

Levy, Sophie (2002). “This Dark Echo Calls Him Home': Writing Father-Daughter Incest Narratives in Canadian Immigrant Fiction", University of Toronto Quarterly: A Canadian J ournal of the Humanities, 71, 4: 864-80.

Loewenstein, J oseph (1984). Reponsive readings: Versions of Echo in Pastoral, Epic, and the J onsonian. New Haven, London: Yale University Press.

Marshall, Gail (1998). Actresses on the Victorian Stage: Feminine Performance and the Galatea Myth. Cambridge: Cambridge University Press.

Mizzau, Marina (1979). Eco e Narciso: Parole e silenzi nel conflitto uomo-donna. Torino: Boringhieri. 
Naik, M. K. (1980). "Echo and Voice in Indian Poetry in English" en Chirantan Kulshrestha (ed.), Contemporary Indian English Verse: An Evaluation. New Delhi: Atlantic Highlands: Arnold-Heinemann; Humanities: 27-40.

O’Donell, Patrick (1997). Echo Chambers: Figuring Voice in Modern Narrative. Iowa City: University of Iowa Press.

Philip, Nourbese Marlene (1997). A Genealogy of Resistance. Toronto: Mercury Press.

Pivato, J oseph (1994). Echo. Essays on other Literatures. Toronto: Guernica.

Raoul, Valerie (1990). "Echo/ Narcissa: Woman as Diarist”, New Comparison: A J ournal of Comparative and General Literary Studies 9:16-27.

Ray, Mohit, K. (ed.), (2007). The Atlantic Companion to Literature in English. New Delhi: Atlantic.

Reid, Jane D. (1993). The Oxford Guide to Classical Mythology in the Arts, 1300-1900s. Oxford University Press: London.

Sage, Lorna (1999). The Cambridge Guide to Women's Writing in English. Cambridge: Cambridge University Press.

Segal, Naomi (1988). Narcissus and Echo: Women and the French Récit. Manchester: Manchester University Press.

- (1989). "Echo and Narcissus", en Teresa Brennan (ed.), Between Feminism and Pscychoanalysis. London: Routledge:168-187.

Spaas, Lieve y Trista Selous (eds.), (2000). Echoes of Narcissus. New York; Oxford: Berghan Books.

Spivak, Gayatri (1993). “Echo”, New Literary History 24, 1: 17-43.

Vinge, Louise (1967). The Narcissus Theme in Western European Literature up to the Early 19th Century. Lund: Gleerup. 